\title{
Epidemia de Clostridium difficile, Chile 2012. Informe de la Sociedad Chilena de Infectología. Una aproximación científico histórica
}

\author{
Felipe Cabello
}

\section{Clostridium difficile epidemic, Chile 2012. Report of the Chilean Society for Infectious Diseases. A scientific historical analysis}

A Summary Report from the Chilean Society for Infectious Diseases regarding the presence of a Clostridium difficile epidemic with several fatalities in Chile's premier emergency public hospital in Santiago is used to make a scientific historical analysis of the situation. This Summary Report identifies several hygienic and sanitary shortcomings that may have played a role in triggering this major epidemic. These include deficiencies in hand washing policies, overcrowding of beds in wards, relaxation of infection control policies, antimicrobial therapy mismanagement and lack of laboratory support. The relevance of these shortcomings to the epidemic is further supported by the lack of any laboratory evidence for the presence of hypertoxigenic strains of $C$. difficile. In an era of whole genome sequencing of pathogens to guide therapy, prevention, and epidemiological studies of infectious diseases, it is illuminating and sobering, as this report so clearly demonstrates, to realize that many epidemics of hospital infections still result from breakdowns in classical and ancillary asepsis and infection control measures developed in the nineteenth century by Semmelweis, Nightingale and Lister. As the Summary Report suggests, such hygienic breakdowns in countries like Chile are usually brought about by lack of implementation and regulation of national hospital infection control policies resulting from the shift of economic resources from the public to the private sector, despite the former being responsible for health care of $80 \%$ of the population.

Key words: Clostridium difficile, epidemics, history of medicine, $19^{\text {th }}$ Century.

Palabras clave: Clostridium difficile, epidemia, historia de la medicina, Siglo diecinueve.

"Postulo mi doctrina para desterrar al terror de las maternidades... El tiempo del silencio ha quedado atrás y tengo el derecho y la obligación de polemizar.”

Ignaz Semmelweis, 1861.

$\mathrm{E}$ n los dos últimos años las técnicas llamadas de tercera generación de secuenciación de ADN, $\checkmark$ que permiten secuenciar repetida y rápidamente el genoma total de patógenos, han producido cambios dramáticos en el estudio de la genética y de la biología molecular de estos microrganismos ${ }^{1,2}$. Estas técnicas comienzan también a aplicarse al estudio de la epidemiología de las enfermedades infecciosas ya sea comunitarias e intrahospitalarias con gran efectividad ${ }^{1-4}$. La aplicación de ellas a patógenos aislados de pacientes y de su ambiente, genera información rápida que es capaz de influenciar el tratamiento clínico de los mismos mediante antimicrobianos y el control de sus infecciones, trazando su fuente de origen y de su diseminación ${ }^{1-4}$. Estas técnicas permiten diferenciar una bacteria de otra con gran fineza y exactitud a nivel de diferencias poliformorficas de una base (SNP) en la secuencia del ADN de diversos genes ${ }^{1-4}$. Ellas permiten además identificar su resistoma (complemento de genes de resistencia a antibacterianos) y su viruloma (complemento de genes de virulencia) $)^{3,4}$, resultando en la construcción de exactos árboles filogenéticos de gran refinamiento que facilitan el estudio del origen y de la diseminación de estos patógenos en diversos ambientes, incluyendo el hospedero ${ }^{1-4}$.

Estas técnicas y estudios han permitido concluir que los patógenos bacterianos, como era de esperar, están en permanente y rápida evolución y cambio de sus propiedades patogénicas y de su resistencia a antimicrobianos; cambios que les permiten adaptarse con gran plasticidad a modificaciones en sus hospederos y en el ambiente ${ }^{3-5}$. Estas técnicas, y una asociada a ellas, la tipificación por secuenciación de múltiples loci genéticos (MLST) han sido aplicada al estudio de la epidemiología de las infecciones por Streptoccocus pneumoniae, Staphylococcus aureus y Clostridium diffícile arrojando novedosos e inesperados resultados $^{3-8}$. Por ejemplo, en el caso de $C$. difficile, una publicación reciente indicaría que en una sala de hospital, las adecuadas medidas de control de infecciones por esta bacteria parecieran ser insuficientes para controlar la diseminación de la infección ${ }^{5,6}$. Esto tal vez porque ellas
Department of Microbiology and Immunology New York Medical College Valhalla, NY 10595, USA.

Recibido: 11 de julio de 2012

Correspondencia a: Felipe Cabello C cabello@nymc.edu 
habrían sido adquiridas antes de la hospitalización a través de los alimentos o habrían sido adquiridas de pacientes en otros lugares del hospital frecuentados por ellos como los servicios radiológicos y endoscópicos ${ }^{5,6,8}$. Se podría pensar que estos nuevos y sorprendentes hallazgos justificarían la prolongada epidemia de infecciones por Clostridium difficile que ha ocurrido recientemente en la Asistencia Pública de Santiago y que provocara varias muertes ${ }^{5-8}$.

Sin embargo, la lectura del Informe de la Sociedad Chilena de Infectología acerca de esta epidemia de $C$. difficile nos revela un estado sanitario en la prevención de infecciones intrahospitalarias y de manejo de la asepsia en este importante recinto hospitalario nacional, que me atrevería a calificar como correspondiente a los de un tiempo previo al de los descubrimientos de Ignác Semmelweis en 1847 y de Florence Nightingale en 1853 $3^{9,11,12}$. Por ejemplo, el informe demuestra que la técnica del lavado de manos que Semmelweis demostrara como tan efectiva para evitar la trasmisión de la fiebre puerperal a las pacientes por médicos y estudiantes de medicina en las maternidades de Viena y Budapest, tenía en la Asistencia Pública de Santiago una implementación limitada e insuficiente ${ }^{7-11}$. También el informe revela que el descubrimiento de F. Nightingale del papel que el hacinamiento juega en facilitar la diseminación de infecciones entre pacientes hospitalizados, era parcialmente ignorado en este hospital ${ }^{9-12}$. Dicho informe indica, además, de manera preliminar, que esta epidemia de $C$. difficile al parecer no se debe a la aparición en Chile de las nuevas variantes hipertoxigénicas de estas bacterias que comprenden el complejo B1/NAP1/027 lo cual indicaría entonces que son los factores del ambiente y del hospedero los responsables de su aparición y de su larvada evolución ${ }^{9,13,14}$.

Recientemente, el profesor de cirugía de la Universidad de Yale y exitoso y original escritor de asuntos médicos, Sherwin B. Nuland, ha escrito una biografía novelada de Semmelweis titulada "La peste de los doctores. Patógenos, fiebre puerperal y la interesante historia de Ignaz Semmelweis”"15. En esta obra Nuland relata que en la población de Viena y entre sus mujeres grávidas existía el conocimiento que si sus partos eran atendidos en la maternidad $\mathrm{N}^{\circ} 1$ del Hospital General sus posibilidades de salir con vida del parto disminuían dramáticamente debido a la fiebre puerperal $^{10,15}$. Esto se debía a que en la Maternidad $N^{0} 1$ trabajaban los médicos y los estudiantes de medicina, que después de hacer autopsias de parturientas muertas el día anterior de fiebre puerperal, atendían a las grávidas recién admitidas, sin lavarse las manos, trasmitiéndoles de esta manera los estreptococos adquiridos de las fallecidas. En la Maternidad $\mathrm{N}^{\circ} 2$ del hospital los partos eran atendidos por matronas las cuales, por no hacer autopsias, eran incapaces de jugar un rol en la trasmisión del estreptococo a las futuras madres ${ }^{10,15}$.

Nuland comienza su obra retratando de manera ma- gistral y trágica el terror de una joven embarazada el año 1847 al saber que había sido asignada a la maternidad No 1 del Hospital General de Viena, ya que con esto ella inmediatamente conjeturó que se podía morir, dado la alta mortalidad post parto por fiebre puerperal en esa maternidad $^{15}$. Al leer este texto, uno no puede dejar de preguntarse si un terror similar no será experimentado por pacientes en Santiago, el año 2012, por tener que recurrir a la Asistencia Pública en vez de a una clínica privada, si es que ellos han leído los diarios y tienen el conocimiento previo que durante su hospitalización pueden terminar siendo víctimas o una fatalidad más de una infección por C. difficile. En Chile existe ahora la posibilidad de hacer la secuencia total del genoma de aislamientos de C. difficile gracias al Centro Nacional de Genómica, Proteómica y Bioinformática, fundado y parcialmente financiado por CONICYT y un consorcio de universidades chilenas y también en el Instituto de Salud Publica.

Sin embargo, es indudable que dado el informe de la Sociedad Chilena de Infectología, lo que la Asistencia Pública, y probablemente varios otros hospitales de Chile necesitan para controlar las infecciones por C. difficile, es implementar rigurosamente las medidas de asepsia y aislamiento introducidas a la practica médica por Semmelweis, Nightingale y Lister en el siglo XIX, más que usar las novedosas técnicas de biología molecular ahora disponibles ${ }^{9}$. Además el informe señala que respecto de esta epidemia también existieron limitaciones en la mantención de la información epidemiológica de los enfermos, en la tabulación de sus datos, en el manejo microbiológico de las muestras clínicas y de aislamientos bacterianos, procedimientos que son fundamentales para obtener la interpretación y los frutos adecuados del uso de las técnicas moleculares que incluyen la secuenciación completa del ADN de genomas de patógenos ${ }^{5-8}$.

Como Nuland lo señala en su libro al titularlo "La peste de los doctores” implicando con ello que la fiebre puerperal era una enfermedad trasmitida por médicos, Semmelweis fue el primer doctor que introdujo el concepto de que los médicos y los estudiantes de medicina, al practicar medicina de una manera inadecuada, son capaces de producir un daño mayor que el problema que tratan de resolver ${ }^{10,11,15}$. Esto resultó en que el reconocimiento de la fiebre puerperal como "una enfermedad transmisible pero no contagiosa” en las palabras de Semmelweis, antes de los descubrimientos de Pasteur y Koch, fuera retrasado por prejuicios basados en la ignorancia y la vanidad profesional ${ }^{10,11,15}$. El reconocimiento de la efectividad del lavado de manos en prevenirla, fue también retardado por varios años debido a la resistencia de los médicos a reconocer que su actividad podría tener aspectos negativos y aun fatales para la salud de sus pacientes ${ }^{10,11,15}$. Este retraso sin dudas generó la muerte innecesaria de miles de mujeres y fue también facilitado por las autoridades 
médicas contemporáneas a Semmelweis en Viena y Budapest, y que de acuerdo al famoso cirujano Theodor Billroth eran "una generación de médicos formados en una restrictiva camisa de fuerza, con anteojos obscuros y algodones en los oídos... que inhibían el pleno uso de sus facultades..."11.

Al continuar leyendo el informe de la Sociedad Chilena de Infectología, pareciera apreciarse tal vez en Chile respecto de esta epidemia de $C$. difficile, una situación similar a la descrita por Billroth en la Viena de hace ya 160 años, ya que en este informe además de los problemas elementales de asepsia, de manejo de antimicrobianos y de aseo, se advierte una inexplicable tardanza de las autoridades responsables en reaccionar frente al problema y a buscar una solución a sus probables causas ${ }^{9,11}$. Esta aparente inercia acerca de la situación pareciera adquirir mayor fuerza al leer un artículo de prensa en el cual autoridades de salud responsables de estos asuntos caracterizan a esta fatal epidemia de $C$. difficile como una anécdota ${ }^{16}$, al mismo tiempo que minimizan su relevancia como una sonora campanada de alarma respecto de la, al parecer decimonónica situación de asepsia y de prevención de las infecciones hospitalaria en los hospitales públicos, que atienden a más de $80 \%$ de la población chilena. La literatura científica también demuestra que ya hace más de doce años atrás diversos investigadores chilenos habían señalado a la aparición de infecciones por C. difficile como un problema emergente en el país ${ }^{17-20}$. Sin embargo, una vez más las autoridades políticas y sanitarias han fallado en responder de una manera veloz y efectiva para evitar el aumento de esta patología, detener la evolución de esta epidemia y atender de esta manera a las necesidades de la mayoría de la población que teóricamente debieran representar y servir ${ }^{21,22}$.

La corrección de situaciones como éstas es fundamental, no sólo para proteger la salud pública del presente sino que también para proteger la salud pública del futuro. Porque la plasticidad del genoma de $C$. difficile dada parcialmente por su habilidad de recombinar genéticamente, demostradas por la técnicas moleculares descritas ${ }^{5-8}$, hace que recintos como la Asistencia Pública de Santiago, con las deficiencias destacadas en el informe, se conviertan en verdaderos reactores que aceleran la evolución de esta bacteria a formas con una mayor resistencia y más virulentas ${ }^{5-8}$. Porque un ambiente con grandes concentraciones del patógeno aumenta las posibilidad de variación genéti- ca producida, ya fuese por mutación y por recombinación como resultado de la transferencia horizontal de genes ${ }^{5-8}$. También está claro que situaciones de este tipo fracasan en ser anécdotas ya que son el resultado de persistentes políticas económicas y de salud pública, incapaces en proveer bienestar a la generalidad de la población que se atiende en los hospitales públicos de Chile. Ellas también exigen a los profesionales de la salud a trabajar en condiciones que quebrantan diariamente un aspecto ético fundamental de sus actividades como es el de "Primun non nocere”. Además, ellas plantean interrogantes acerca de la calidad de la educación médica que puede ser impartida en estas condiciones de incumplimiento de los cánones científicos y éticos básicos de estas profesiones y sin que exista un reconocimiento generalizado de su presencia y una constante crítica constructiva para corregirlas.

El Informe señala que "La conjunción de pacientes debilitados, hacinamiento hospitalario, deficiencias en los procesos de atención del paciente incluyendo el consumo excesivo de antibióticos y la contratación de empresas externas de aseo y limpieza son hechos comunes en el sistema público de salud del país ${ }^{9 ”}$. Como ya tan bien lo apuntara el joven Rudolf Virchow a mediados del siglo XIX en su famoso estudio de las causas de la epidemia de tifus de la Silesia Superior (Alta) en Alemania, y como al parecer lo recogen hoy en día las demandas de nuestros estudiantes, la solución de estos problemas necesita de “más democracia, cultura y prosperidad, ya que todos los individuos tienen derecho a la salud y el Estado deber hacerse responsable de que esto ocurra adecuada y equitativamente"23.

Agradecimientos. Agradezco el estimulo para este análisis a los anónimos, dedicados y hábiles profesionales que redactaron el Informe de la Sociedad Chilena de Infectología y al apoyo que ésta prestara a su actividad. Lo dedico a la memoria de mis profesores de la Escuela de Medicina de la Universidad de Chile que de manera infatigable, sin claudicación y por siete años me enseñaron dos condiciones que considero esenciales para la práctica profesional científica y ética, desentrañar la realidad individual y colectiva y actuar para mejorarla, cumpliendo así con el Juramento Hipocrático. Especialmente al Profesor Amador Neghme a quien escuchara por primera vez hablar de Ignaz Semmelweis. Agradezco a la Dra. Katia Velásquez M. y a un revisor anónimo acertadas sugerencias en la presentación del texto.

\section{References}

1.- Dolgin E. Sequencing of superbugs seen as key to combating their spread. Special Report on Pathogen Genomics. Nature Med 2010; 16: 1054.

2.- Otto T D. Real-time sequencing. Nature Rev
Microbiol 2011; 9: 633.

3.- Croucher N J, Harris S R, Fraser C, Quail M A, Burton J, van der Linden M, et al. Rapid pneumococcal evolution in response to clinical interventions. Science 2011; 331: 430-4.

4.- $\quad$ Koser C U, Holden M T G, Ellington M J,
Cartwright E J P, Brown N M, Ogilvy-Stuart A $\mathrm{L}$, et al. Rapid whole-genome sequencing for investigation of a neonatal MRSA outbreak. N Engl J Med 2012; 366: 2267-75.

5.- Walker A S, Eyre D W, Wyllie D H, Dingle K E, Harding R M, O’Connor L, et al. Characterization of Clostridium difficile hospital 
ward-based transmission using extensive epidemiological data and molecular typing. PLoS Medicine 2012; 9: e101172.

6.- Harbarth S, Samore M H. Clostridium: Transmission difficile? PLoS Medicine 2012; 9: e1001171.

7.- He M, Sebaihia M, Lawley T D, Stabler R A, Dawson L F, Martin M J, et al. Evolution dynamics of Clostridium difficile over short and long time scales. Proc Natl Acad Sci USA 2010; 107: 7527-32.

8.- $\quad$ Stabler R A, Dawson L F, Valiente E, Cairns M D, Martin M J, Donahue E H, et al. Macro and micro diversity of Clostridium difficile isolates from diverse sources and geographical locations. PLoS One 2012; 7: e31559.

9.- Resumen del Informe sobre sobre las infecciones por Clostridium difficile en hospital de urgencia Asistencia Publica Dr. Alejandro del Rio. Sociedad Chilena de Infectologia, Santiago, Mayo 2012.

10.- Semmelweis I. The etiology, concept and prophylaxis of child bed fever (Excerpts). Classics in Social Medicine. Social Medicine 2008; 3: 4-12.

11.- Nuland S B. The germ theory before germs.
The enigma of Ignác Semmelweis. In Doctors. The Biography of Medicine. Alfred A Knopf Inc (New York); 1988. p. 228.

12.- Williams H. Florence Nightingale. The Healing Touch. Charles C Thomas (Springfield, IL) 1951; p. 158.

13.- Bartlett J G. Clostridium difficile: progress and challenges. Ann N Y Acad Sci 2010; 1213: 62-9.

14.- Carroll K C, Bartlett J G. Biology of Clostridium difficile: Implications for epidemiology and diagnosis. Ann Rev Microbiol 2011; 65: 501-21.

15.- Nuland S B. The Doctors'Plague. Germs, Childbed Fever, and the Strange Story of Ignác Semmelweis. WW Norton \& Company (New York); 2003.

16.- Director del Servicio de Salud Central califica como "una anécdota” casos de Clostridium en Posta. La Tercera. Junio 16, 2012.

17.- Balassiano I T, Yates E A, Domingues R M C P, Ferreira E O. Clostridium difficile: a problem of concern in developed countries and still a mystery in Latin America. J Med Microbiol 2012; 61: 169-79.

18.- Álvarez L M, Robinson, González L, Briceño
L I, Cofré D C, Labarca L J, Vial C P, et al. Clinical diagnosis of Clostridium difficile diarrhea. Rev Med Chil 2001; 129: no. 6. http:// www.scielo.cl/scielo.php?script=sci_arttext\&pid $=$ S0034-98872001000600004.

19.- Gardilcic F M, Fica C A, Chang R M, Llanos M C, Luzoro V A. Clostridium difficile associated diarrhea in an adults hospital. A descriptive study. Rev Chilena Infectol 2000; 17: 307-12.

20.- Herrera R P, Cotera F A, Fica C A, Galdo A T, Alvo A M. High frequency of Clostridium difficile diarrhea among patients with renal diseases. Rev Med Chil 2003; 131: 397-403.

21.- Cabello F C. Epidemiology and laboratories. The missing link on the plans to study and prevent infectious diseases in Chile. Rev Chilena Infectol 2008; 25: 14-6.

22.- Cabello FC. The roles that the environment, the sanitary conditions, the epidemiology and vaccines play in the challenge of infectious diseases. Rev Chilena Infectol. 2010; 27: 565-9.

23.- Nuland S B. The fundamental units of life. Sick cells, microscopes and Rudolf Virchow. In Doctors. The Biography of Medicine. Alfred A Knopf Inc (New York); 1988. p. 304. 